\title{
3D Guide Wire Navigation from Single Plane Fluoroscopic Images in Abdominal Catheterizations
}

\author{
Martin Groher ${ }^{2}$, Frederik Bender ${ }^{1}$, Ali Khamene ${ }^{3}$, Wolfgang Wein ${ }^{3}$, \\ Tim Hauke Heibel ${ }^{2}$, Nassir $\mathrm{Navab}^{2}$ \\ ${ }^{1}$ Siemens AG Healthcare Sector AX, Forchheim, Germany \\ ${ }^{2}$ Computer Aided Medical Procedures (CAMP), TUM, Munich, Germany \\ ${ }^{3}$ Siemens Corporate Research (SCR), Princeton, NJ, USA \\ groher@cs.tum.edu
}

\begin{abstract}
Nowadays, abdominal catheterizations are mainly guided by 2D fluoroscopic imaging from one view, which complicates this task due to missing depth information, vessel invisibility, and patient motion. We propose a new technique for 3D navigation of guide wires based on singleplane 2D fluoroscopic views and a previously extracted 3D model of the vasculature. In order to relate the guide wire, which is only visible in the fluoroscopic view, to the $3 \mathrm{D}$ vasculature, we comprise methods for $2 \mathrm{D}-3 \mathrm{D}$ registration, apparent breathing motion compensation, guide wire detection, and robust backprojection. With this $2 \mathrm{D}$-to-3D transfer, the navigation can be performed from arbitrary viewing angles, disconnected from the static perspective view of the fluoroscopic sequence.
\end{abstract}

\section{Introduction}

Minimally invasive catheter-guided interventions are common practice in modern hospitals and are usually guided by fluoroscopic imaging from one view. A guide wire can be visualized thus, which is moving in the image sequence due to respiratory patient motion and the physician's push/pull actions. In order to visualize blood vessels, which cannot be seen in fluoroscopic X-ray views, most hospitals acquire a high-resolution 2D Digitally Subtracted Angiogram (DSA) to avoid blind navigation. Using 2D DSAs, the navigation is still difficult at vessel overlays produced by the projection, or at vessel segments that run perpendicular to the image plane. Thus, 3D angiographic data were introduced into interventional workflow. However, they must be related to the current fluoroscopic frame, which is currently done via user interactions, hampering the navigation process. In order to relate $3 \mathrm{D}$ high resolution data to the current 2D fluoroscopic frame, systems have been proposed based on 2D-3D registration [1], or C-arm calibration [2]. Atasoy et al. [3] also takes breathing motion into account, which is common in abdominal fluoroscopic sequences, by compensating for the apparent motion of the guide wire. While clearly beneficial in terms of vessel visibility, depth perception cannot be increased with these approaches. 
We propose a method for $3 \mathrm{D}$ guide wire navigation that transfers the $2 \mathrm{D}$ navigational procedure to $3 \mathrm{D}$ by taking information about $2 \mathrm{D}$ guide wire tip location, apparent breathing motion, view and 3D vessel geometry into account (Fig. 1). From a vascular model extracted from a 3D scan, and a DSA, we compute the projection matrix of the C-arm device. We relate the DSA to the current fluoroscopic frame by tracking the apparent catheter motion in 2D. From possible guide wire tip locations in $2 \mathrm{D}$, we determine candidate $3 \mathrm{D}$ locations via backprojection to the $3 \mathrm{D}$ vessel model. With the estimates of view geometry and motion, we derive uncertainties for all these locations and determine the most probable of all 3D candidates using a variable bandwidth kernel estimator for information fusion. With our proposed system, which has been recently presented in [4], we successfully bring abdominal guide wire navigation to 3D, thus solving the problems of blind navigation and lack of depth perception.

\section{Method}

\subsection{Data and preprocessing}

The data sets that form the basis to our method are a fluoroscopic image sequence $\mathcal{S}$, a high resolution DSA with the same projection parameters as the fluoroscopic sequence, and a 3D angiographic scan from which we extract a $3 \mathrm{D}$ vessel model $\mathcal{V}$ as described in [5]. To determine the $3 \mathrm{D}$ position $\mathbf{X}$ of the guide wire tip, we need to know its position $\mathbf{X}$ within the current 2D fluoroscopic image frame

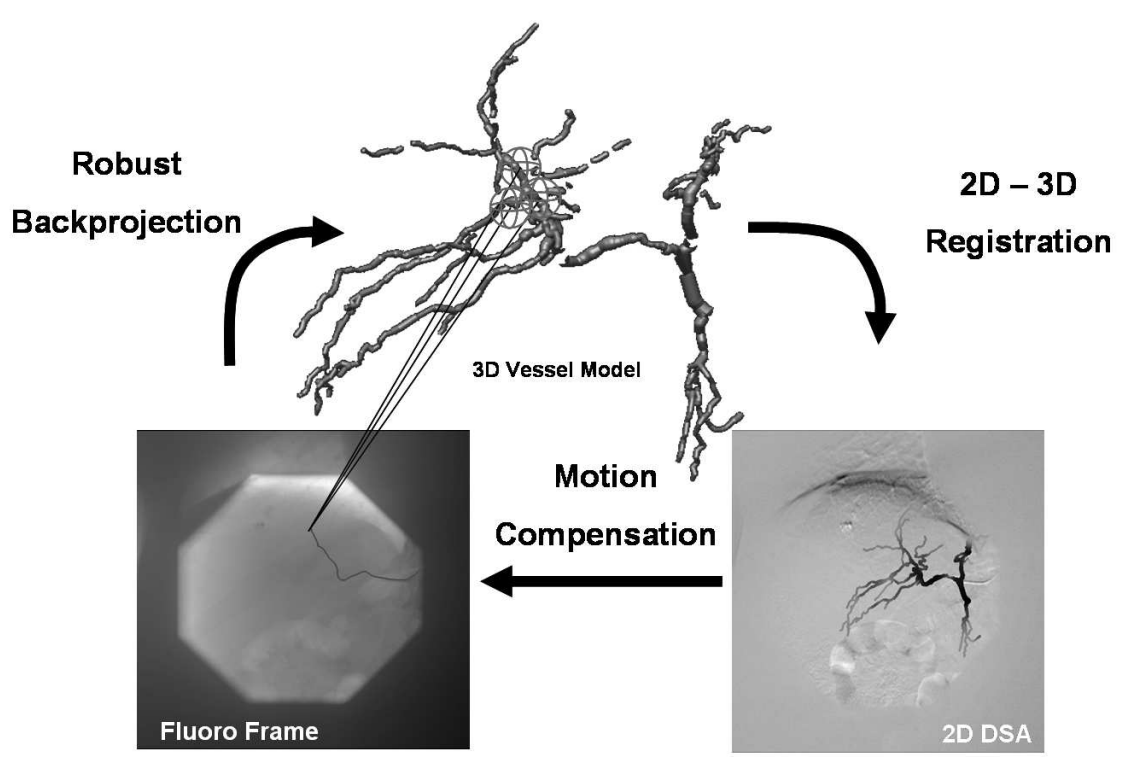

Fig. 1. 3D guide wire navigation 
$\mathcal{S}_{i}$. To this end we employ a line detector proposed in [6]. We need a projection matrix $\mathrm{P}$, which aligns the $3 \mathrm{D}$ scan with the DSA image. A method, which is robust to deformation changes and different vessel contrast as proposed in [1] is used here. To countervail apparent respiratory motion, we compute the $2 \mathrm{D}$ motion in-between frames using the template based approach proposed in [3]. This method yields a global 2D translation, which approximates the 2D apparent breathing motion and relates each fluoroscopic frame to the initial DSA.

\subsection{D guide wire tip localization}

Our method fuses all gathered information to compute the 3D correspondence of the guide wire tip location for every image frame, assuming a given uncertainty connected to each input parameter. In a first step, we subtract the respiratory motion displacement of the current image frame from the guide wire tip location to obtain the tip location $\mathbf{X}_{0}$ with respect to the DSA image. This is advantageous, since we can then use the pseudo-inverse of $\mathrm{P}$ to backproject the guide wire tip into 3D space. However, a simple backprojection of $\mathbf{X}_{0}$ will not always yield a satisfactory solution. First, ambiguities can arise if the backprojection ray intersects the vessel model multiple times. Second, the estimate of the guide wire tip location in 2D is prone to error caused by detection and motion compensation. We solve these problems with a statistical approach that is based on the creation of possible 2D guide wire tip candidates and the evaluation of their registration accuracy.

We take errors from detection and apparent motion estimation into account by randomly modifying the computed displacements in a range of $[ \pm 10, \pm 10]$ pixels to generate $n 2 \mathrm{D}$ tip candidates around $\mathbf{X}_{0}$, denoted by $\mathbf{X}_{k}$ where $k=1 . . n$. We backproject each $\mathbf{X}_{k}$ using the pseudo-inverse of $\mathrm{P}$, compute the closest point on the vessel model $\mathcal{V}$ to the resulting ray, and define it to be a 3D correspondence candidate $\mathbf{X}_{k}$. Next, we compute an uncertainty connected to each $\mathbf{X}_{k}$ that is introduced by the uncertainty of the pose parameters $\Theta$ from P. We randomly modify the registered pose $\Theta \mathrm{m}$ times within an interval of $[ \pm 5, \pm 5] \mathrm{mm}$ for the translational, and $[ \pm 5, \pm 5]^{\circ}$ for the rotational parameters. This results in $m$ different poses $\Theta_{j}$, which yield $n$ sets of $m 3$ D points: $\left\{\mathbf{X}_{k_{j}}\right\}, k=1 . . n, j=1 . . m$.

We assign a weight $r_{j}$ to each $\mathbf{X}_{k_{j}}$, where $r_{j}$ is computed as the reciprocal of the cost function used for 2D-3D registration [1], evaluated for pose $\Theta_{j}$, and normalized such that $r_{j} \in[0 ; 1]$. The uncertainty of each $\mathbf{X}_{k}$ can be represented as its weighted covariance matrix (visualized by green spheres in Fig. 1)

$$
C_{k}=\frac{1}{\sum_{j=1}^{m} r_{j}} \sum_{j=1}^{m} r_{j}\left(\mathbf{X}_{k_{j}}-\overline{\mathbf{X}}_{k}\right)\left(\mathbf{X}_{k_{j}}-\overline{\mathbf{X}}_{k}\right)^{T}
$$

where $\overline{\mathbf{X}}_{k}=\frac{1}{\sum_{j=1}^{m} r_{j}} \sum_{j=1}^{m} r_{j} \mathbf{X}_{k_{j}}$ is the weighted mean.

In a final step, we fuse this uncertainty information to obtain an estimate for the 3D guide wire tip location. An approach to fuse such information is 
Table 1. Results of target errors $\epsilon$ and standard deviations $\sigma$ in $\mathrm{mm}$

\begin{tabular}{lrcccccccc}
\hline Phantom (Fig. 3(c)) & $\epsilon_{x}$ & $\epsilon_{y}$ & $\epsilon_{z}$ & $\epsilon$ & $\sigma_{x}$ & $\sigma_{y}$ & $\sigma_{z}$ & $\sigma$ \\
\hline Case 1 & New & 1.78 & 2.51 & 1.96 & $\mathbf{3 . 9 8}$ & 1.55 & 1.00 & 1.10 & $\mathbf{1 . 4 4}$ \\
(light gray) & Naïve & 2.70 & 4.97 & 24.39 & 26.59 & 1.38 & 2.94 & 43.41 & 42.58 \\
Case 2 & New & 0.89 & 1.90 & 1.13 & $\mathbf{2 . 6 6}$ & 0.71 & 1.74 & 0.67 & $\mathbf{1 . 5 8}$ \\
(dark gray) & Naïve & 1.35 & 2.71 & 19.38 & 20.75 & 1.59 & 3.82 & 37.27 & 36.87 \\
\hline
\end{tabular}

the Variable-Bandwidth Density-Based Fusion algorithm (VBDF), proposed by Comaniciu in [7]. It is robust to outliers, which occur as 3D correspondence candidates on a wrong vessel branch in our scenario, and involves the uncertainties of the measurements. In this approach, we estimate a density function from all measurement means $\overline{\mathbf{X}}_{k}$ and define its most significant mode to be the estimated 3D guide wire tip location $\mathbf{X}$. We involve information about the 3D location that was computed from the previous fluoroscopic image frame. The idea is to weigh the measurements $\overline{\mathbf{X}}_{k}$ dependent on their distance to the estimate of the previous frame. This is justified by the fact, that the guide wire will probably not move dramatically between successive image frames. To ensure the $3 \mathrm{D}$ guide wire to be inside a vessel, we pick the closest point on $\mathcal{V}$ to the computed $\mathbf{X}$ and define this point to be the final 3D guide wire tip position.

\section{Experiments and results}

To test our method, we ran a study on a realistic breathing phantom with a known ground truth. To this end we use a phantom including a vessel system, which is formed by a set of connected plastic tubes fixed in liquid wax (Fig. 3(a)). The breathing motion is simulated by an electronic pump that is attached to the phantom, yielding apparent in-plane displacements of up to 14 pixels (in 1024 $\times 1024$ images). The image data is acquired with a Siemens AXIOM Artis $\mathrm{dBA}$, a calibrated bi-plane C-arm device. It allows us to grab two fluoroscopic sequences (Fig. 3(c)) from which we can compute the guide wire tip's 3D ground truth position. The vessel model $\mathcal{V}$ is extracted from a rotational run under contrast agent. For testing our algorithm, we used only one of the projection sequences, where two particular overlay cases are evaluated (Fig. 3(b)). The DSA for registration was created from a second, contrasted sequence. 3D Euclidean errors and associated standard deviations are shown in Tab. 1. A comparison is made with a naïve approach, which does not use uncertainty information for backprojection. Please note that the error especially in ray direction $\left(\varepsilon_{z}\right)$ can be reduced significantly using our method.

\section{Conclusion}

In this work we propose an algorithm, which robustly facilitates 3D navigation of guide wires in abdominal catheterizations using fluoroscopic images from a 
Fig. 2. (a) Breathing phantom. (b) Rendered 3D volume of phantom with arrows marking the path of case 1 (light gray), case 2 (dark gray). (c) Phantom fluoroscopy showing tubes and guide wire. The circels mark the same paths as arrows in (b).

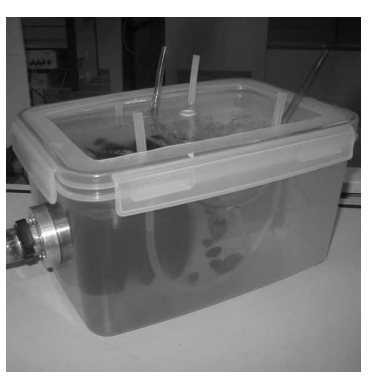

(a)

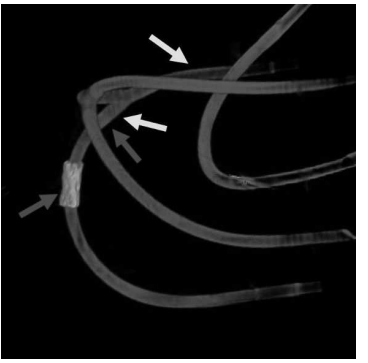

(b)

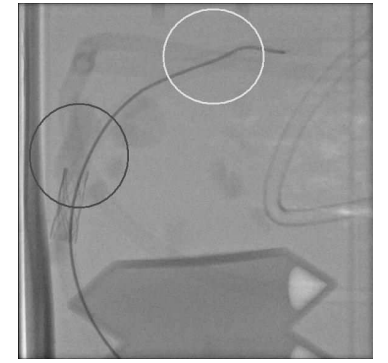

(c)

monoplane C-arm system. Encouraged by the good results from synthetic and phantom tests, we will proceed with an extensive patient study. Further ideas are aiming at a feedback system for physicians in cases where the C-arm configuration is not optimal in regard to the desired operation situs, or where the system's predicition is too ambiguous. The concepts and information presented in this paper are based on research and are not commercially available.

\section{References}

1. Groher M, Bender F, Hoffmann RT, et al. Segmentation-driven 2D-3D registration for abdominal catheter interventions. Procs MICCAI. 2007; p. 527-535.

2. Gorges S, Kerrien E, Berger MO, et al. Model of a vascular C-arm for 3D augmented fluoroscopy in interventional radiology. Procs MICCAI. 2005; p. 214-222.

3. Atasoy S, Groher M, Zikic D, et al. Real-Time respiratory motion tracking: Roadmap correction for hepatic artery catheterizations. Procs SPIE. 2008.

4. Bender F, Groher M, Khamene A, et al. 3D dynamic roadmapping for abdominal catheterizations. MICCAI. 2008.

5. Selle D, Preim B, Schenk A, et al. Analysis of vasculature for liver surgery planning. IEEE Tran Med Imaging. 2002;21(8):1344-1357.

6. Steger C. An unbiased detector of curvilinear structures. IEEE Trans Pattern Anal Mach Intell. 1998;20(2):113-125.

7. Comaniciu D. Nonparametric information fusion for motion estimation. Procs IEEE CVPR. 2003; p. I-59 - I-66. 